\title{
BABE is A Beautiful Science: Why is it Not More Often Used in Oncology? Retsky $\mathbf{M}^{*}$ \\ Harvard T.H. Chan School of Public Health, Boston, MA 02115, USA and University College London, UK
}

This document is based on a presentation I made at a BABE conference in Baltimore, MD in October 2014. I am Editor in Chief of Journal of Bioequivalence and Bioavailability (BABE) and my field of research is oncology. Many papers cross my desk in process of review and publication in our journal. Why is it, I frequently ask myself, that few if any papers that use bioequivalence and bioavailability as a method to test and ultimately provide new drugs are in the oncology field? On the surface, this seems incongruous. BABE is a beautiful science so it would seem to be natural to use this technology to make new drugs available in a multibillion dollar market. Instead, clinical trials are used in oncology to determine if new products are as good as or better than old products. That is an expensive and time consuming process. Breast cancer for example often takes over a decade to run its course from diagnosis to death or certain cure. From my perspective, this is probably the major reason why, if we are winning the war on cancer, it is not happing rapidly.

That long time scale is daunting to pharmaceutical companies that naturally want to get return on investment in reasonable time. Typically then their focus is on drugs that address metastatic disease. For that late stage category, the disease runs its course for better or worse in months or at most a few years.

The answer that always comes to mind is that we don't yet understand the disease process sufficiently to use BABE. As readers of this journal will know, in order to use BABE, one has to know in some detail how the disease progresses and how $\operatorname{drug} A$ works so he or she can determine without needing a clinical trial if $\operatorname{drug} B$ will work as well. The plain and simple unfortunate explanation is that we don't yet sufficiently understand the relapse process to use BABE in general oncology.

But I am here to report what may be some progress. It is well known that much research and development is occurring in methods to prevent or delay death in late stage disease where metastatic tumor is present and threatening life. I am confident they will ultimately be successful but the therapies will very likely be toxic, resistance will appear, and be far too expensive for all but the developed countries. What I am telling you now is we may be making progress in learning how most relapses occur and perhaps how to prevent that from happening. A general solution that would prevent relapses is a main unsolved problem in oncology. When someone is first diagnosed, at least in developed countries, the disease has rarely shown evidence of metastatic activity. This is called early stage disease. If a way could be found to more often prevent relapse from early to late stage that would be a major and welcome development.

For a number of years, my colleagues and I have been studying an unexpected bimodal relapse pattern in breast cancer. There are many relapses that sharply appear in the first 3 years after surgery. Then there is a minimum at about 4 years and then surprisingly starting at the 5 th year there is a broad wave of relapses extending out to 15 or so years. This was unexpected but by now we have identified this pattern in over 20 independent breast cancer databases from US, Europe and Asia. The quantity of relapses varies with the ordinary prognostic factors such as age, receptors, tumor size and the presence of lymph nodes with cancer cells. However the timing of the relapse peaks does not change with these prognostic factors. Using computer simulation, we came to the conclusion that the early waves of relapses (which constitute the bulk of relapses in absence of therapy) were triggered somehow or other by something that happened at or about the time of surgery. We were able to explain many clinical features of breast cancer with this hypothesis.

We had a few ideas on what caused the early relapses and how to prevent them but nothing was dramatic enough to gather too much interest from funding agencies or trial groups. However a new and unexpected process became apparent when a paper was published in 2010 by a Brussels anesthesiology group. They reported in a retrospective study that a common inexpensive NSAID analgesic drug given perioperatively seemed to reduce the relapses in months $9-18$ by five-fold. Teaming up with the Brussels group, we came to the idea that perhaps surgery produces a transient period of systemic inflammation. That could initiate a number of mechanisms that could result in tumor outgrowth. As one simple example, inflammation can be accompanied by capillary leakage which in the presence of circulating cancer cells could cause the cancer cells to be trapped in distant organs. Metastatic tumors would then show up in 1-3 years corresponding to the early peak in relapse data [1].

Much has been published, there has been some publicity [2], a small trial is underway in Brussels, and a clinical trial is planned for Seoul, South Korea. We must wait and see if it will reduce relapses and lead to new understanding of early relapses that can be studied with BABE technology.

Disclosure: Michael Retsky is on the board of directors of the Colon Cancer Alliance (www.ccalliance.org) and has a patent pending for treatment of early stage cancer. No other conflicts of interest to report.

\section{References}

1. Retsky M, Demicheli R, Hrushesky WJ, Forget P, De Kock M, et al. (2013) Reduction of breast cancer relapses with perioperative non-steroidal antiinflammatory drugs: new findings and a review. Curr Med Chem 20: 4163-4176.

2. http://www.propublica.org/article/where-are-the-low-cost-cancer-treatments?

*Corresponding author: Retsky M, Harvard TH Chan School of Public Health, Boston, MA 02115, USA and University College London, UK, Tel: 617-432-3472 E-mail: michael.retsky@gmail.com

Received February 19, 2015; Accepted February 20, 2015; Published February 28, 2015

Citation: Retsky M (2015) BABE is A Beautiful Science: Why is it Not More Often Used in Oncology? J Bioequiv Availab 7: e63. doi:10.4172/jbb.10000e63

Copyright: (c) 2015 Retsky M. This is an open-access article distributed under the terms of the Creative Commons Attribution License, which permits unrestricted use, distribution, and reproduction in any medium, provided the original author and source are credited. 